\title{
An Exploratory Study of the Relationships between Narcissism, Self-Esteem and Instagram Use
}

\author{
Olga Paramboukis, Jason Skues, Lisa Wise \\ Department of Psychological Sciences, Faculty of Health, Arts and Design, Swinburne University of Technology, \\ Hawthorn, Australia \\ Email: Iwise@swin.edu.au
}

Received 21 March 2016; accepted 25 April 2016; published 28 April 2016

Copyright (C) 2016 by authors and Scientific Research Publishing Inc.

This work is licensed under the Creative Commons Attribution International License (CC BY).

http://creativecommons.org/licenses/by/4.0/

(c) (i) Open Access

\begin{abstract}
The aim of this mixed-methods exploratory study was to examine the relationship between narcissism, self-esteem and Instagram usage and was motivated by unsubstantiated media claims of increasing narcissism due to excessive use of social networks. A sample of 200 participants responded to an online survey which consisted of the Five Factor Narcissism Inventory (FFNI), the Rosenberg Self-Esteem scale, and the Instagram Usage, Behaviours, and Affective Responses Questionnaire (IUBARQ) constructed specifically for the purposes of this study. There was only weak evidence for any relationship between narcissism and Instagram usage, suggesting that media concerns are somewhat exaggerated. However the negative correlation between vulnerable narcissism and self-esteem warrants further examination.
\end{abstract}

\section{Keywords}

Narcissism, Vulnerable Narcissism, Grandiose Narcissism, Instagram, Self-Esteem, Social Media

\section{Narcissism, Self-Esteem and Social Media}

Recent research has suggested that young people today are more narcissistic compared to previous generations [1]. This statistical increase in scores on narcissism measures has coincided with the introduction, uptake, and widespread use of social networking sites such as Facebook and Twitter. Several researchers have investigated the simultaneous increase in narcissism and social media use and note that self-reported narcissism tends to be associated with different motivations and patterns of usage for social media [2]-[10]. Yet few studies have investigated whether social networking sites other than Facebook or Twitter are related to high levels of self- 
reported narcissism. This study contributes to the current research by investigating how Instagram, a social networking site focusing on editing, posting, and commenting on images, is associated with the personality trait of narcissism.

\subsection{Measures of Narcissism}

Although narcissism has been investigated for around 40 years (see [11] for a review), there is still ongoing debate about whether narcissism should be conceptualised as a psychiatrically diagnosed personality disorder or a subclinical personality trait [12]-[14]. Narcissism has been viewed from a social and personality psychology perspective as a trait comprising multiple dimensions shaped from the earlier clinical construct. A common distinction in both the clinical and social/personality psychology literature is between grandiose and vulnerable narcissism [15]. The grandiose dimension refers to traits such as exhibitionism, callousness, extraversion, manipulativeness, superiority, aggression, indifference and seeking of acclaim, whereas the vulnerable dimension is believed to reflect feelings of inadequacy, emptiness and shame, reactive anger, helplessness, hypervigilance to insult, excessive shyness and interpersonal avoidance [16] [17]. In general, more emphasis has been placed on the grandiose aspect of narcissism compared to the vulnerable aspect. The current study defines narcissism from the social and personality perspective as a sub-clinical trait with two factors, grandiose and vulnerable narcissism respectively.

\subsection{Narcissism and Self-Esteem}

Some researchers have posited significant conceptual overlap between narcissism and self-esteem with individuals high in both traits having a higher opinion of themselves [18]-[20]. In contrast, others have argued that, while self-esteem is considered to be an intrapersonal trait, narcissism is primarily interpersonal [21]. Narcissistic individuals may present a false mask of high self-esteem, scoring high on explicit measures of self-esteem, but showing much lower scores on implicit measures of the same trait [21].

Another possible explanation for the equivocal findings relates to the notion of different aspects of the self, e.g., agentic versus communal self-views [18] [19]. Campbell et al. [19] found that narcissists and people with high self-esteem report positive, albeit distinct, self-views. That is, narcissists perceive themselves as better than average primarily on traits reflecting agency (e.g., competence), whereas individuals with high self-esteem hold superior beliefs regarding both agency and communal traits. In this regard, the self-regulatory strategies employed by narcissists involve seeking attention and admiration by comparing themselves to others, and by defending their competence to others. Given the multiple alternative explanations relating to the relationship between narcissism and self-esteem, this study will contribute to this research by exploring the relationship between these two constructs in the context of social network use.

\subsection{Narcissism and Social Networking Sites}

Several researchers have investigated the relationship between narcissism and social media with studies ranging from testing simple correlations between narcissism scores and basic usage and descriptive data, to studies that have examined how different dimensions of narcissism relate to motivations and behaviours associated with different social network sites including MySpace, Facebook, and Twitter. Not surprisingly, the findings have been mixed. The early studies focused primarily on Facebook and reported significant correlations between narcissism and time per day spent using Facebook, number of Facebook friends, numbers of photos and the selection of specific profile photos, and status updates [3] [8]. Bergman et al. [2] did not find narcissism to be related to actual social media usage, but instead that it was positively associated with motivations such as wanting to have a lot of online friends, believing others are interested in what they are doing, and wanting to show others what they were doing. This highlights that researchers need to look beyond simplistic quantitative variables in relation to social media use.

In one of the first studies to separate narcissism into different factors and relate these factors to Facebook use, Carpenter [4] found Grandiose-Exhibitionism to be associated with self-promoting behaviours, number of Facebook friends, seeking social support and retaliating against perceived mean comments, while Entitlement/ Exploitativeness was related to more anti-social behaviours such as retaliation and checking up on whether he or she is being talked about by others. 
Panek et al. [10] also examined how different aspects of narcissism were related to Facebook and Twitter use in a study of both undergraduate students and adults. For university students, exhibitionism was related to time spent and number of Facebook status posts, and entitlement was related to time spent per day. For adults, superiority and authority were related to Facebook checking, and vanity was associated with Facebook posting and Twitter checking. According to Panek et al., university students use Twitter as a "technologically augmented megaphone" that allows them to demonstrate their superiority to others. For adults, it appears to be Facebook that is used in this manner. Davenport et al. [5] also examined the role of narcissism and motives in relation to Facebook and Twitter in university and adult samples. Similarly to Panek et al. [10], Twitter was found to be associated with narcissistic university students for whom tweeting was the preferred mode of communication. However, it was more important for narcissists in both samples to have more Facebook friends than Twitter followers, which is probably a reflection of the different affordances and relationships with people in the audience for both of the social network sites. Ong et al. [9] also found in their sample of secondary school students that after controlling for extraversion, narcissism was positively associated with self-generated content on Facebook (e.g., profile picture selection etc), but not system-generated content (e.g., number of friends or photos etc). However, Ong et al. acknowledged that they did not consider privacy settings, which can limit the size and intended audience.

It may be that the expression of narcissistic behaviour through social networking sites is more of a by-product of a society that is becoming increasingly more "self-centred”, and social media merely provide another arena in which narcissistic tendencies can be displayed. Alternatively, social media may facilitate, encourage and applaud narcissistic behaviours in a problematic spiral that magnifies the degree of narcissism even further. A further possibility is that this association is merely the result of changes in the way people respond to narcissism and self-esteem scale items on the respective scales (i.e., with less false modesty than in previous generations), rather than a actual change in the nature of the personality traits themselves.

\subsection{Instagram}

Instagram is a photo and video sharing social networking site that is becoming increasingly popular among young people ${ }^{1}$. Instagram prompts users to edit photos using inbuilt, easily-applied filters and special effects, before posting these images onto the Instagram site [22]. Instagram differs from Facebook and Twitter through being entirely focused on images. According to Instagram Press [22], 300 million of its users have an Instagram account that they regularly use (monthly). There is also an average of 70 million photos being posted daily worldwide, attracting 2.5 billion "likes" [22]. Nonetheless, in spite of its widespread usage and specific focus on posting images, there is a dearth of research on Instagram, and how it relates to narcissism.

Based on the integration of previous research on other social networking sites and the affordances that Instagram provides to users, it is argued that narcissistic tendencies such as attention-seeking and exhibitionism may be facilitated by Instagram usage due to its specific image-based applications and functions. Firstly, Instagram facilitates the selection and editing of photos that can be used to make a specific impression to others by glamorizing their portrayal of themselves or their lives. Such behaviour aligns with grandiose narcissism traits such as attention-seeking, vanity, self-promotion and exhibitionism. Secondly, "liking” and “commenting” functions are available on Instagram for followers and do not require the formation of a deeper relationship (which may be achieved via instant messaging functions). This aspect of the site may greatly appeal to highly narcissistic individuals (both grandiose and vulnerable) as they tend to not retain close relationships despite their desire for social contact [23] [24]. Thirdly, "hash tagging” may also be used as a form of self-promotion by both highly vulnerable and grandiose narcissistic individuals as a user may choose to hash tag their photo with popular search terms with the intention of their photo being seen by a larger audience.

\subsection{The Current Study}

The current study aims to explore the relationships between narcissism and its subtypes, self-esteem, and Instagram use. The main motivation for the study was to investigate the relationship between narcissism and social

\footnotetext{
${ }^{1}$ Although typically considered a separate social networking site in its own right, it is important to highlight that Instagram is often linked to Facebook and Twitter, which means that content and communication on these sites is not necessarily mutually exclusive and will depend on whether one has linked the respective profiles. Indeed since this study was undertaken, Facebook has purchased Instagram, making the boundary between them even more difficult to define.
} 
media use by targeting a social networking site (i.e., Instagram) that specifically facilitates the type of behaviour that has been shown to be associated with narcissism in previous research (i.e., photo-sharing).

H1: Individuals who score high on narcissism will engage in more Instagram behaviours.

We anticipate that some aspects of narcissism may be related to self-esteem and that both narcissism and self-esteem may influence patterns of Instagram use. These relationships are best investigated as research questions, in keeping with the exploratory nature of the study.

RQ1: How do different aspects of narcissism relate to self-esteem and Instagram use?

$\boldsymbol{R Q 2 :}$ Is there a difference in the pattern of Instagram use for individuals who are classified as grandiose narcissists compared to those classified as vulnerable narcissists?

\section{Method}

\subsection{Participants}

After preliminary data screening, during which participants with missing data were deleted listwise, a total of 200 participants completed the study. There were 148 female and 52 male participants, with ages ranging from 18 to $51(\mathrm{M}=22.41$, Med $=21, \mathrm{SD}=6.15)$. Of these participants, only 154 had Instagram accounts, and the majority of data will be reported from the demographic of interest, constituting 141 of the 154 Instagram users who were under the age of 26 .

\subsection{Measures}

Five Factor Narcissism Inventory (FFNI [25]). The Five Factor Narcissism Inventory is a 148-item narcissism personality trait measure that was designed from a theoretical framework that views narcissistic traits as maladaptive extensions of traits from the Five Factor Model of personality. The FFNI contains 15 different facets which form two subtypes of narcissism, namely Grandiose Narcissism (Indifference, Exhibitionism, Thrill Seeking, Authoritativeness, Grandiose Fantasies, Manipulativeness, Exploitativeness, Entitlement, Arrogance, Lack of Empathy and Acclaim Seeking) and Vulnerable Narcissism (Reactive Anger, Shame, Need for Admiration and Cynicism/Distrust). Participants were asked to respond on a five point Likert scale ranging from (1) Strongly Disagree to (5) Strongly Agree with higher scores corresponding to more of a particular trait.

Rosenberg Self-Esteem Scale (RSS [26]). Participants completed the Rosenberg self-esteem scale which is a measure of global self-esteem that consists of 10 items which are measured on a four point Likert scale ranging from (1) Strongly Disagree to (4) Strongly Agree [26].

Instagram Usage, Behaviour, and Emotional Reactions Questionnaire (IUBRQ). Participants completed the Instagram Usage, Behaviour, and Emotional Reactions Questionnaire (IUBRQ), which was designed for the purpose of the current study. Since no specific scale is currently available to operationalise Instagram usage, the following questionnaire was created to investigate only the areas of interest in this study, which included frequency of usage, frequency of Instagram-specific behaviours and the attitudes and affective reactions towards Instagram usage. The selection of the content for this scale was formed via informal focus groups with a small cohort of research students from our laboratory, along observations of online forums regarding behaviours on Instagram undertaken by the first author.

Instagram Usage. This section comprised 12 questions ranging from open-ended estimates of time or frequency to yes/no responses or 4-point likert scale responses.

Instagram Behaviours. This section consisted of 16 questions relating to ways of interacting with Instagram. Participants responded on a five point scale $(1=$ Never; 2 = Rarely; 3 = Sometimes, 4 = Often; and 5 = Very Often) based on their recollection of activities over the past month.

Instagram Attitudes. This section consisted of 5 questions relating to motivations for interacting with Instagram. Participants responded on a four point scale $(1=$ Not at all important; 2 = Slightly important; 3 = Kind of important; and 4 = Very important).

Instagram Emotional Reactions. The section consisted of 3 open-ended questions where participants were invited to respond to questions about how they characterised some aspects of Instagram usage, and also how they emotionally reacted to positive and potentially negative feedback they may receive on Instagram posts.

\subsection{Procedure}

Participants were invited to complete a 30-minute online survey which consisted of the FFNI, the RSES, and the 
IBURQ. Participants completed the survey at a time and location that was convenient to them, and they were provided with a debriefing statement immediately upon the completion of the survey. The study was approved by Swinburne University's ethical review committee. Data were analysed using Microsoft Excel, SPSS Version 23.0, and R Version 3.2.3.

\section{Results and Discussion}

\subsection{Instagram and Other Social Networks}

Of the 200 participants recruited for the study, 154 had Instagram accounts, of whom 122 were female and 32 were male. Of the 154 participants with Instagram accounts, 62 participants linked their Instagram account to another social network. Of these, 53 linked their Instagram account to Facebook, 9 to Twitter, 8 to Tumblr. Some linked to more than one social network, with 8 linking to both Facebook and Twitter, 6 linking to both Facebook and Tumbler, 3 linking to both Twitter and Tumblr. No participants linked to all three. Thirty six of the remaining 46 non-Instagram users reported having a Facebook account, and only 5 did not report any use of social networking sites at all.

Within our sample, it appeared that Facebook was still the most popular social networking site, and that Instagram was often used in conjunction with other social media sites. Since our data were collected for this study, Facebook has purchased Instagram, underscoring the transient nature of usage/behaviour patterns for specific social network sites.

\subsection{Instagram Privacy Settings}

We asked participants three questions relating to privacy: 1) was their account publically available?; 2) did they accept follower requests from people unknown to them?; and 3) did their bio page contain personal information? (see Table 1). While it might be expected that users would maintain consistency between different privacy settings (for example, keeping their content private, accepting follower requests only from people they know and restricting personal information in their bio), this was not always the case. For example, as can be seen from Table 1, 24 participants who kept their accounts private accepted follower requests from strangers. The inconsistency in privacy settings is more likely to reflect a lack of knowledge of account settings and how they operate than any deliberate strategy for information dissemination.

\subsection{Instagram Usage, Behaviours and Emotional Reactions Questionnaire}

The vast majority of the sample of Instagram users were university students under the age of 26 (141 of 154 Instagram users) and this was the demographic targeted for our analysis of relationships between personality traits and Instagram behaviours and attitudes. The IUBRQ survey attempted to quantify aspects of Instagram usage in terms of frequency of interaction with Instagram, the types of behaviours engaged in through Instagram, and the motives and attitudes surrounding Instagram interactions.

\subsection{Instagram Usage}

While half of the participants (51\%) visited their Instagram site often or very often, the majority of the sample posted photos occasionally or rarely (77\%). This suggests that the majority of Instagram users are consumers rather than creators of content.

Table 1. Privacy settings on Instagram: cross-tabulation of participants who accept followers who are unknown to them, have personal information in their bio sections and have their accounts publically available.

\begin{tabular}{ccccc}
\hline \multirow{2}{*}{ Account Public } & \multicolumn{2}{c}{ Accept Unknown } & \multicolumn{2}{c}{ Bio with Personal Info } \\
\cline { 2 - 5 } & Yes & No & Yes & No \\
\hline Yes & 69 & 13 & 33 & 49 \\
No & 24 & 48 & 19 & 53 \\
\hline
\end{tabular}




\subsection{Instagram Behaviours}

The Behaviours section of the IUBRQ survey addressed ways in which users could interact via Instagram and reflects features of Instagram at the time when the research was undertaken.

As can be seen from the top panel of Figure 1, most participants rarely used the Instagram features listed, consistent with finding that very few Instagram users regularly post photos. The most frequent behaviour was use of hashtags, which was the only listed behaviour that the majority of Instagram users performed at least sometimes.

In order to generate possible metrics to capture the degree of interaction with Instagram, a Principal Components Analysis was conducted on the 16 Instagram Behaviour items of the IUBRQ. A one-factor solution was revealed to be the best fitting model in which 13 of the 16 items had primary loadings above 0.3 on a single factor and explained $25 \%$ of the variance. On the basis of this analysis, we compiled a single Instagram Behaviours score from all the items of this section for use in the correlational analyses with personality traits.

\subsection{Instagram Attitudes}

The Attitudes section of the IUBRQ survey addressed attitudes towards Instagram usage. As can be seen from the bottom panel of Figure 1, being portrayed and recognised in a positive light was important to the majority of participants. However it should be noted that positive acclaim was not purely reflected through getting "likes", something that $35 \%$ of the participants did not find particularly important. Achieving symmetry in the layout of images on the screen was also not particularly important to most participants.

\subsection{Narcissism, Self-Esteem and Instagram Behaviours}

In order to test whether narcissism relates to self-esteem and Instagram use, summary data were first calculated for each of the narcissism and self-esteem scales and relevant subscales used in this study and reported in Table 2.

Narcissism. The median values for both grandiose and vulnerable narcissism scores were approximately at the midpoint of the scoring, with some participants scoring toward the upper limit of possible scores. At the trait level, the highest-scoring trait was "acclaim seeking” $(M=36.33, S D=6.85)$, whilst the lowest scoring trait was

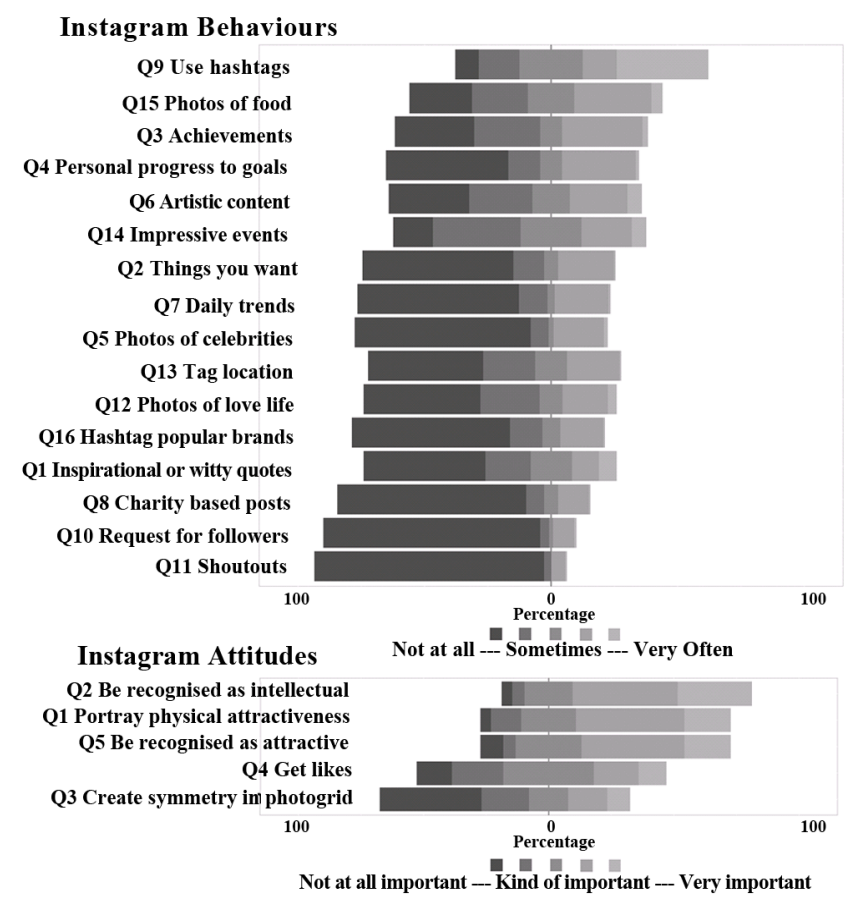

Figure 1. Likert scales for each item on the Instagram Behaviours and Instagram Attitudes sections of the IUBARQ. 
Table 2. Means, SDs, medians and ranges for scales and subscales from the FFNI and the Rosenberg Self-Esteem Scale.

\begin{tabular}{|c|c|c|c|c|}
\hline \multirow{2}{*}{ Personality variable } & \multicolumn{4}{|c|}{ Descriptive Statistics } \\
\hline & Mean & $\mathrm{SD}$ & Median & Range \\
\hline Indifference & 26.90 & 8.55 & 26 & $10-49$ \\
\hline Exhibitionism & 32.35 & 6.81 & 33 & $15-49$ \\
\hline Thrill seeking & 21.13 & 6.87 & 21 & $8-36$ \\
\hline Arrogance & 22.10 & 6.19 & 21 & $10-48$ \\
\hline Entitlement & 20.23 & 5.41 & 20 & $10-48$ \\
\hline Manipulativeness & 24.80 & 7.16 & 23 & $10-47$ \\
\hline Exploitativeness & 20.94 & 7.29 & 20 & $10-47$ \\
\hline Authoritativeness & 31.99 & 7.62 & 33 & $10-50$ \\
\hline Grandiose fantasies & 31.44 & 7.02 & 32 & $11-49$ \\
\hline Lack of empathy & 17.72 & 5.22 & 17 & $10-42$ \\
\hline Acclaim seeking & 36.33 & 6.85 & 37 & $11-50$ \\
\hline Grandiose Total & 285.80 & & 283 & $155-462$ \\
\hline Reactive anger & 27.53 & 6.56 & 28 & $11-46$ \\
\hline Shame & 31.78 & 8.12 & 32 & $12-50$ \\
\hline Need for admiration & 28.39 & 6.22 & 28 & $10-44$ \\
\hline Distrust & 26.46 & 5.72 & 27 & $12-39$ \\
\hline Vulnerable Total & 114.20 & & 116 & $51-168$ \\
\hline FFNI Total & 400.00 & & 401 & $269-622$ \\
\hline Rosenberg Self-Esteem & 29.33 & 5.34 & 29 & $11-39$ \\
\hline
\end{tabular}

"lack of empathy" $(M=17.72, S D=5.22)$.

Self-esteem. Self-esteem scores were found to be high in the sample with more than $75 \%$ of the sample scoring more than the midpoint of the scale.

Correlations. Spearman's rank order correlations were used to test the significance of associations between narcissism, self-esteem, Instagram Behaviours as measured by a composite score from the IUBRQ Behaviours items and Instagram Attitudes as measured by a composite score from the IUBRQ Attitudes items. As can be seen in Figure 2, overall narcissism (FFNI Total) did not correlate with self-esteem (RSS). However when narcissism was separated into grandiose and vulnerable dimensions, a weak positive correlation was found between grandiose narcissism and self-esteem $(\rho=0.35, p<0.001)$, whilst a negative moderate strength correlation was found between vulnerable narcissism and self-esteem $(\rho=-0.59, p<0.001)$. That is, those Instagram users with higher levels of grandiose narcissism tended to report higher self-esteem levels, whilst vulnerable narcissists reported lower self-esteem levels. All three narcissism scores were positively associated with both Instagram Attitudes and Instagram Behaviours, but the relationships between self-esteem and both Instagram Attitudes and Instagram Behaviours were not significant.

An inspection of the item level data for selected Instagram behaviours revealed significant correlations between grandiose narcissism and Instagram behaviours such as "Hashtagging popular or expensive brands" $(\rho=$ $0.29, p<0.001)$, "Posting photos of things you want, but do not have" $(\rho=0.19, p<0.001)$, "Posting photos of celebrities or people you admire" $(\rho=0.18, p<0.05)$, and "Posting photos of progress towards physical health, fitness and wellbeing" $(\rho=0.20, p<0.05)$. Moreover, vulnerable narcissism correlated with "Hashtagging popular or expensive brands $(\rho=0.24, p<0.001)$, "Posting photos of things you want, but do not have" ( $\rho=$ $0.21, p<0.05)$, "Posting photos of celebrities or people you admire" $(\rho=0.34, p<0.001)$, "Posting photos of yourself at impressive events or functions" ( $\rho=0.23, p<0.001)$, and "Request for followers" (e.g. \#follow4follow) 


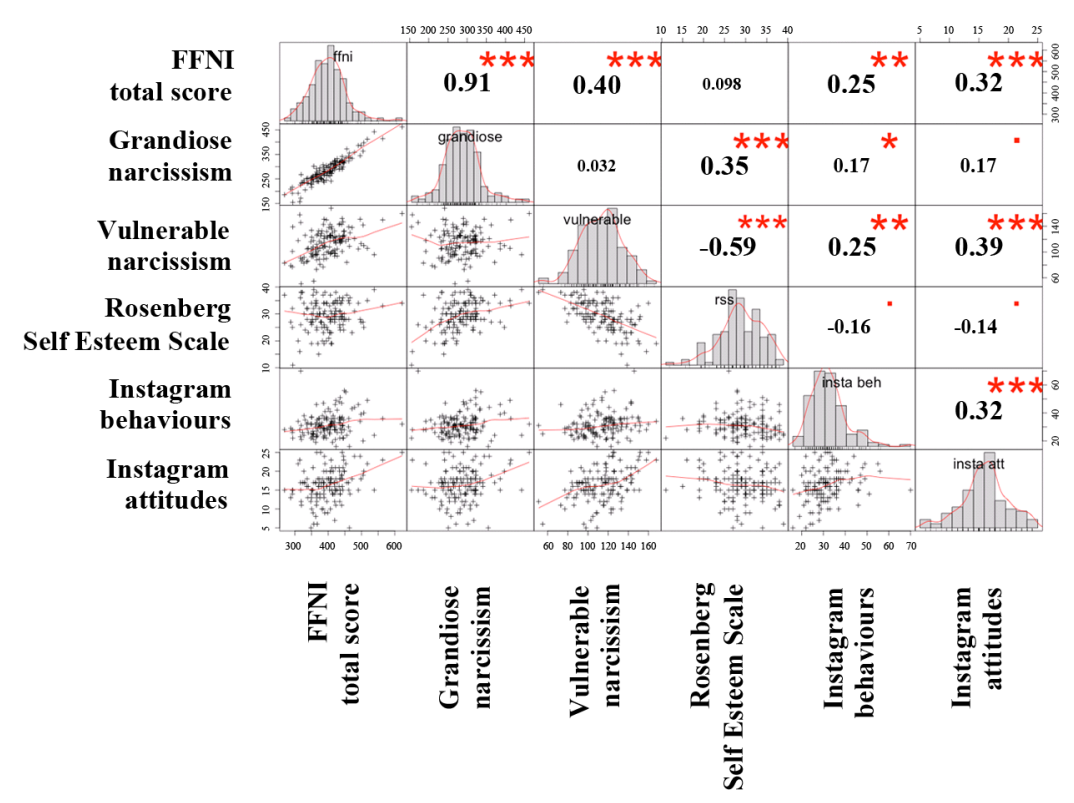

Figure 2. Scatterplots, frequency histograms and correlations for narcissism scores, self-esteem scores, Instagram Behaviours and Instagram Attitudes. Ranges of values for scatterplots and histograms can be identified from the range column of Table 2.

$(\rho=0.17, p<0.05$ ). For the most part, the pattern of correlations were similar for grandiose and vulnerable narcissism, with the only differences being a significant association between grandiose narcissism and posting photos of physical health, fitness and wellbeing, and a significant relationship between vulnerable narcissism and requests for followers.

\subsection{Qualitative Analyses}

The similarities and differences between grandiose and vulnerable narcissism were also explored using openended questions exploring emotional reactions to feedback received from other people in response to a participant's Instagram behaviours. Prior to performing a content analysis on the open-ended responses, three groups of participants were identified according to their combined Grandiose and Vulnerable Narcissism scores, namely 1) a High Grandiose/Low Vulnerable group; 2) a High Vulnerable/Low Grandiose; group; and 3) a High Grandiose/High Vulnerable group. Responses to three questions were compared across the three groups. The first question was 1) "Do you feel happy or elated when you receive a lot of feedback on a post?" The most common response to this question across all three groups was "Yes, because it gives a sense of validation and/or approval”. According to one participant, “...I feel that people are 'liking' an aspect of my life and further more are showing approval of me as a person”.

In response to the second question, "If your post receives no feedback, how does that make you feel?", participants classified in the high grandiose groups typically responded with indifference, whilst the most common responses for participants in the high vulnerable/low grandiose group were experienced negative emotions (32.1\%) and deleted the post (17.9\%).

For the last question, "If someone is critical of your post, how does that make you feel?", the most common response for the high vulnerable, low grandiose group was "Block/Delete the post and/or the person whom was critical” (28.6\%), with the second most common response relating to feeling defensive (25\%). Indeed, one participant states "I react negatively and defensively. It makes me feel judged and unintelligent". In contrast, both high grandiose groups tended to be neutral in their responses.

In sum, the content analysis revealed that participants who were simultaneously high in vulnerable narcissism and low in grandiose narcissism reacted more strongly to both negative and positive interactions on Instagram in comparison to participants who scored either high on grandiose narcissism exclusively or scored high on both grandiose and vulnerable narcissism. 


\section{General Discussion}

This study aimed to explore the interrelationships between narcissism, self-esteem and Instagram usage. Although there were differences in how grandiose and vulnerable narcissism related to self-esteem, neither types of narcissism were strongly associated with Instagram usage as identified via the IUBRQ.

\subsection{Narcissism and Self-Esteem}

The FFNI total score for narcissism was not correlated with self-esteem, however vulnerable narcissism was negatively correlated with self-esteem, while grandiose narcissism was positively correlated with self-esteem, albeit at a lower level. This finding provides further evidence for the distinction between grandiose and vulnerable narcissism and supports the view that self-esteem is an important factor that distinguishes between those subtypes of grandiose and vulnerable narcissism (see [18] [19]). Moreover the data suggest that vulnerable narcissism may have more of an intrapersonal component compared to grandiose narcissism, and that internal feelings of inadequacy and helplessness are more closely related to self-esteem compared to feelings of superiority over others. However, it still remains unclear whether those with high grandiose narcissism levels do in fact have higher self-esteem levels, or whether their underlying need for superiority and exhibitionism results in presenting an overly inflated self view on a self-report measure of self-esteem.

\subsection{Narcissism and Instagram Behaviours}

Vulnerable narcissism also had the highest degree of correlation with both Instagram Behaviours and Instagram Attitudes although these relationships were not strong. In terms of specific Instagram behaviours, posting up photos of one's physical appearance was more associated with grandiose narcissism, while requests for followers was more associated with vulnerable narcissism. The qualitative data identified those who were high in vulnerable but low in grandiose narcissism showed stronger emotional reactions to Instagram feedback. These findings are broadly consistent with past research that suggests that narcissism is related to self-generated content on social network sites, rather than system-generated content [9]. Those high on vulnerable narcissism appeared to be more interested in increasing their popularity and seeking the approval of others, whereas grandiose narcissists used overt attempts of drawing attention to themselves in order to be admired. That vulnerable narcissists use Instagram as a platform to seek out positive feedback aligns with the notion that people seek validation from others in order to help boost self-esteem [27]. This would also help to explain the strong emotional reactions to negative feedback for individuals high on vulnerable narcissism, which includes the dimensions of reactive anger, need for admiration and shame. In contrast, grandiose narcissists appear to seek out opportunities to engage in behaviours that afford self-promotion (see [4] [6]) in order to maintain their elevated positive selfview [3], which fits with the grandiose dimensions of exhibitionism and seeking of acclaim.

\subsection{Implications}

The findings from this study have provided theoretical, methodological and practical implications. Firstly, this study provides further evidence for distinguishing between grandiose and vulnerable narcissism, and that these two subtypes are differentially related to self-esteem. Secondly, this study has expanded the research on narcissism subtypes and social network use, targeting a social network that specifically focuses on posting images. However despite this specific focus, the study has shown only weak evidence for any relationship between narcissism and Instagram use. This study also developed a survey that aimed to quantify specific Instagram behaviours in an attempt to uncover more nuanced patterns of behaviour relating both to reasons for posting content, and reactions to feedback on content. Even with detailed quantitative and qualitative data on Instagram usage, the weak evidence for any association between narcissism and Instagram suggests that Instagram offers a platform for expression of existing narcissistic tendencies rather than a medium that encourages extremes of narcissistic behaviour in people who do not normally show such tendencies.

\subsection{Limitations and Future Research}

Although this study was one of the very studies to have attempted to operationalise Instagram behaviours by developing a specific measure that quantifies some of the common Instagram behaviours, it should be noted that 
the frequency of particular Instagram behaviours may not accurately reflect the importance placed on these behaviours by the individual participants. The qualitative data collected in this study provided some insights into the way individual participants interpret and react to Instagram content but did not really provide a coherent narrative on why people use Instagram, nor is it clear that such a narrative exists at a psychological level (i.e., at a deeper level than that it offers an easy way to share photos with friends and family).

It is important to note that this study targeted Instagram for its specific emphasis on posting and sharing photos rather than on providing instant messaging for communicating with others. However our data suggest that Instagram is often used in conjunction with other social network sites and most participants had more followers on Facebook than on Instagram. The fact that Facebook has now purchased Instagram may result in a tighter integration of Instagram features into Facebook, or a change in Instagram features that renders some items of the IUBRQ irrelevant. Indeed the primary limitation of most studies on social networking sites is that they only provide a transient snapshot of behaviour patterns as both the technologies and user bases evolve.

\subsection{Conclusion}

In conclusion, the primary finding from this study is that there is only weak evidence for any relationship between narcissism and Instagram usage, suggesting that media concerns about social media giving rise to unprecedented narcissistic behaviour are somewhat exaggerated. However, it appears that there is a complex relationship between narcissism and self-esteem such that vulnerable narcissism is negatively correlated with selfesteem and grandiose narcissism is positively associated with self-esteem albeit more weakly. Vulnerable narcissism appears to be more strongly associated with Instagram usage, with vulnerable narcissists seeking acclaim and being more sensitive to feedback on their posts. In contrast grandiose narcissists appear to use Instagram to exhibit their superiority over others but are not overly sensitive to feedback. Despite the limitations of the study, the findings have provided a better understanding of the associations between narcissism subtypes, self-esteem and Instagram use, and highlight the need for further exploration of the relationship between selfesteem and vulnerable narcissism, which has typically received less attention by researchers than grandiose narcissism.

\section{References}

[1] Twenge, J.M., Konrath, S., Foster, J.D., Campbell, W.K. and Bushman, B.J. (2008) Egos Inflating over TIme: A Cross Temporal Meta-Analysis of the Narcissistic Personality Inventory. Journal of Personality, 76, 875-902. http://dx.doi.org/10.1111/j.1467-6494.2008.00507.x

[2] Bergman, S., Fearrington, M.E., Davenport, S.W. and Bergman, J.Z. (2011) Millenials, Narcissism and Social Networking: What Narcissists do on Social Networks and Why. Personality and Individual Differences, 50, 706-711. http://dx.doi.org/10.1016/j.paid.2010.12.022

[3] Buffardi, L.E. and Campbell, W.K. (2008) Narcissism and Social Networking Web Sites. Personality and Social Psychology Bulletin, 34, 1303-1314. http://dx.doi.org/10.1177/0146167208320061

[4] Carpenter, C.J. (2012) Narcissism on Facebook: Self-Promotional and Anti-Social Behaviour. Personality and Individual Differences, 52, 482-486. http://dx.doi.org/10.1016/j.paid.2011.11.011

[5] Davenport, S.W., Bergman, S.M., Bergman, J.Z. and Fearrington, M.E. (2014) Twitter versus Facebook: Exploring the Role of Narcissism in Motives and Usage of Different Social Media Platforms. Computers in Human Behaviour, 32, 212-220. http://dx.doi.org/10.1016/j.chb.2013.12.011

[6] Fox, J. and Rooney, M.C. (2015) The Dark Triad and Trait Self-Objectification as Predictors of Men's Use and SelfPresentation Behaviours on Social Networking Sites. Personality and Individual Differences, 76, 161-165. http://dx.doi.org/10.1016/j.paid.2014.12.017

[7] Gentile, B., Twenge, J.M., Freeman, E.C. and Campbell, W.K. (2012) The Effect of Social Networking Websites on Positive Self-Views: An Experimental Investigation. Computers in Human Behaviour, 28, 1929-1933. http://dx.doi.org/10.1016/j.chb.2012.05.012

[8] Mehdizadeh, S. (2010) Self-Presentation 2.0: Narcissism and Self-Esteem on Facebook. Cyberpsychology, Behaviour and Social Networking, 13, 357-364. http://dx.doi.org/10.1089/cyber.2009.0257

[9] Ong, E.Y.L., Ang, R.P., Ho, J.C.M., Lim, J.C.Y., Goh, D.H. and Lee, C.S. (2011) Narcissism, Extraversion and Adolescents' Self-Presentation on Facebook. Personality and Individual Differences, 50, 180-185. http://dx.doi.org/10.1016/j.paid.2010.09.022 
[10] Panek, E.T., Nardis, Y. and Konrath, S. (2013) Mirror or Megaphone?: How Relationships between Narcissism and Social Networking Site Use Differ on Facebook and Twitter. Computers in Human Behaviour, 29, 2004-2012. http://dx.doi.org/10.1016/j.chb.2013.04.012

[11] Cain, N.M., Pincus, A.L. and Ansell, E.B. (2008) Narcissism at the Crossroads: Phenotypic Description of Pathological Narcissism across Clinical Theory, Social/Personality Psychology and Psychiatric Diagnosis. Clinical Psychology Review, 28, 638-656. http://dx.doi.org/10.1016/j.cpr.2007.09.006

[12] Kohut, H. (1971) The Analysis of the Self: A Systematic Psychoanalytic Approach to the Treatment of Narcissistic Personality Disorders. International Press, New York.

[13] Kernberg, O.F., (1984) Severe Personality Disorders: Psychotherapeutic Strategies. Yale University, New Haven.

[14] Kernberg, O.F. (1985) Borderline Conditions and Pathological Narcissism. Rowman and Littlefield, Lanham, MD.

[15] Pincus, A.L, and Lukowitsky, M.R. (2010) Pathological Narcissism and Narcissistic Personality Disorder. Annual Review of Clinical Psychology, 6, 421-446. http://dx.doi.org/10.1146/annurev.clinpsy.121208.131215

[16] Ackerman, R.A., Witt, E.A., Donnellan, M.B., Tzresniewski, K.H., Robbins, R.W. and Kashy, D.A. (2011) What Does the Narcissistic Personality Inventory Really Measure? Assessment, 18, 67-87. http://dx.doi.org/10.1177/1073191110382845

[17] Miller, J.D. and Campbell, K. (2008) Comparing Clinical and Social Personality Conceptualizations of Narcissism. Journal of Personality, 76, 449-476. http://dx.doi.org/10.1111/j.1467-6494.2008.00492.x

[18] Bosson, J.K., Lakey, C. E., Campbell, W.K., Zeigler-Hill, V., Jordan, C.H. and Kernis, M.H. (2008) Untangling the Links between Narcissism and Self-Esteem: A Theoretical and Empirical Review. Personality and Social Psychology Compass, 2, 1415-1439. http://dx.doi.org/10.1111/j.1751-9004.2008.00089.x

[19] Campbell, W.K., Rudich, E.A. and Sedikides, C. (2002) Narcissism, Self-Esteem, and the Positivity of Self-Views: Two Portraits of Self-Love. Personality and Social Psychology Bulletin, 28, 358-368. http://dx.doi.org/10.1177/0146167202286007

[20] Hovrath, S. and Morf, C.C. (2010) To Be Grandiose or Not To Be Worthless: Different Routes to Self-Enhancement for Narcissism and Self-Esteem. Journal of Research in Personality, 44, 585-592. http://dx.doi.org/10.1016/j.jrp.2010.07.002

[21] Zeigler-Hill, V. (2006) Discrepancies between Implicit and Explicit Self-Esteem: Implications for Narcissism and SelfEsteem Instability. Journal of Personality, 74, 119-144. http://dx.doi.org/10.1111/j.1467-6494.2005.00371.x

[22] Instagram Press (2015, $13^{\text {th }}$ of January) Retrieved from http://Instagram.com/press/

[23] Kealy, D. and Rasmussen, B. (2012) Veiled and Vulnerable: The Other Side of Grandiose Narcissism. Clinical Social Work Journal, 40, 356-365. http://dx.doi.org/10.1007/s10615-011-0370-1

[24] McGregor, I., Nail, P.R., Kocalar, D. and Haji, R. (2013) I'm OK, I'm OK: Praise Makes Narcissists with Low Implicit Self-Esteem Indifferent to the Suffering of Others. Personality and Individual Differences, 55, 655-659. http://dx.doi.org/10.1016/j.paid.2013.05.007

[25] Glover, N., Miller, J.D., Lynam, D.R., Crego, C. and Widiger, T.A. (2012) The Five-Factor Narcissism Inventory: A Five-Factor Measure of Narcissistic Personality Traits. Journal of Personality Assessment, 94, 500-512. http://dx.doi.org/10.1080/00223891.2012.670680

[26] Rosenberg, M. (1965) Society and the Adolescent Self-image. Princeton University Press, Princeton, NJ.

[27] Krämer, N.C. and Winter, S. (2008) Impression Management 2.0: The Relationship of Self-Esteem, Extraversion, SelfEfficacy, and Self-Presentation within Social Networking Sites. Journal of Media Psychology, 20, 106-116. http://dx.doi.org/10.1027/1864-1105.20.3.106 